\title{
Intrauterine Delivery System Dosage
} Form

National Cancer Institute

\section{Source}

National Cancer Institute. Intrauterine Delivery System Dosage Form. NCI Thesaurus.

Code C149614.

Solid single-dose delivery system intended for intrauterine use that releases its contents of active substance(s) over an extended period of time. 